\title{
AN EXPLICIT FORMULA FOR THE NATURAL AND CONFORMALLY INVARIANT QUANTIZATION
}

\author{
F. RADOUX
}

\begin{abstract}
In [5], P. Lecomte conjectured the existence of a natural and conformally invariant quantization. In [7, we gave a proof of this theorem thanks to the theory of Cartan connections. In this paper, we give an explicit formula for the natural and conformally invariant quantization of trace-free symbols thanks to the method used in [7] and to tools already used in 8 in the projective setting. This formula is extremely similar to the one giving the natural and projectively invariant quantization in $[8]$.
\end{abstract}

\section{INTRODUCTION}

A quantization can be defined as a linear bijection from the space $\mathcal{S}(M)$ of symmetric contravariant tensor fields on a manifold $M$ (also called the space of Symbols) to the space $\mathcal{D}_{\frac{1}{2}}(M)$ of differential operators acting between half-densities.

It is known that there is no natural quantization procedure. In other words, the spaces of symbols and of differential operators are not isomorphic as representations of $\operatorname{Diff}(M)$.

The idea of equivariant quantization, introduced by P. Lecomte and V. Ovsienko in [6] is to reduce the group of local diffeomorphisms in the following way : if a Lie group $G$ acts (locally) on a manifold $M$, the action can be lifted to tensor fields and to differential operators and symbols. A $G$-equivariant quantization is then a quantization that exchanges the actions of $G$ on symbols and differential operators.

In [2], the authors considered the group $S O(p+1, q+1)$ acting on the space $\mathbb{R}^{p+q}$ or on a manifold endowed with a flat pseudo-conformal structure of signature $(p, q)$. They showed the existence and uniqueness of a $S O(p+$ $1, q+1)$-equivariant quantization in non-critical situations.

The problem of the $s o(p+1, q+1)$-equivariant quantization on $\mathbb{R}^{m}$ has a counterpart on an arbitrary manifold $M$. In [5], P. Lecomte conjectured the existence of a natural and conformally invariant quantization, i.e. a quantization procedure depending on a pseudo-riemannian metric, that would be

Date: November 21, 2018.

University of Liège, Institute of mathematics, Grande Traverse, 12 - B37, B-4000 Liège, Belgium email : Fabian.Radoux@ulg.ac.be MSC : 53B10, 53C10, 22E46. 
natural (in all arguments) and that would be left invariant by a conformal change of metric.

We proved in [7] the existence of such a quantization using Cartan connections theory.

The goal of this paper is to obtain an explicit formula on $M$ for the natural and conformally invariant quantization of trace-free symbols. This task can be realized using tools exposed in [7] and [1].

The paper is organized as follows. In the first section, we recall briefly the notions exposed in [7] necessary to understand the article. In the second part, we calculate the explicit formula giving the natural and conformally invariant quantization for trace-free symbols on the Cartan fiber bundle using the method exposed in [7]. In the third section, we develop as in [8] this formula in terms of natural operators on the base manifold $M$, using tools explained in [1], in order to obtain the announced explicit formula. It constitutes the generalization to any weight of density of the formula given by M. Eastwood in [3] thanks to a completely different method. Moreover, Eastwood formula is given under a different form.

\section{Fundamental tools}

Throughout this work, we let $M$ be a smooth manifold of dimension $m \geq 3$.

2.1. Tensor densities. Denote by $\Delta^{\lambda}\left(\mathbb{R}^{m}\right)$ the one dimensional representation of $G L(m, \mathbb{R})$ given by

$$
\rho(A) e=|\operatorname{det} A|^{-\lambda} e, \quad \forall A \in G L(m, \mathbb{R}), \forall e \in \Delta^{\lambda}\left(\mathbb{R}^{m}\right) .
$$

The vector bundle of $\lambda$-densities is then defined by

$$
P^{1} M \times{ }_{\rho} \Delta^{\lambda}\left(\mathbb{R}^{m}\right) \rightarrow M,
$$

where $P^{1} M$ is the linear frame bundle of $M$.

Recall that the space $\mathcal{F}_{\lambda}(M)$ of smooth sections of this bundle, the space of $\lambda$-densities, can be identified with the space $C^{\infty}\left(P^{1} M, \Delta^{\lambda}\left(\mathbb{R}^{m}\right)\right)_{G L(m, \mathbb{R})}$ of functions $f$ such that

$$
f(u A)=\rho\left(A^{-1}\right) f(u) \quad \forall u \in P^{1} M, \forall A \in G L(m, \mathbb{R}) .
$$

2.2. Differential operators and symbols. As usual, we denote by $\mathcal{D}_{\lambda, \mu}(M)$ the space of differential operators from $\mathcal{F}_{\lambda}(M)$ to $\mathcal{F}_{\mu}(M)$.

The space $\mathcal{D}_{\lambda, \mu}$ is filtered by the order of differential operators. The space of symbols is then the associated graded space of $\mathcal{D}_{\lambda, \mu}$. It is also known that the principal operators $\sigma_{l}(l \in \mathbb{N})$ allow to identify the space of symbols with the space of contravariant symmetric tensor fields with coefficients in $\delta$-densities where $\delta=\mu-\lambda$ is the shift value.

More precisely, we denote by $S_{\delta}^{l}\left(\mathbb{R}^{m}\right)$ or simply $S_{\delta}^{l}$ the space $S^{l} \mathbb{R}^{m} \otimes$ $\Delta^{\delta}\left(\mathbb{R}^{m}\right)$ endowed with the natural representation $\rho$ of $G L(m, \mathbb{R})$. Then the 
vector bundle of symbols of degree $l$ is

$$
P^{1} M \times_{\rho} S_{\delta}^{l}\left(\mathbb{R}^{m}\right) \rightarrow M .
$$

The space $\mathcal{S}_{\delta}^{l}(M)$ of symbols of degree $l$ is then the space of smooth sections of this bundle, which can be identified with $C^{\infty}\left(P^{1} M, S_{\delta}^{l}\left(\mathbb{R}^{m}\right)\right)_{G L(m, \mathbb{R})}$. Finally, the whole space of symbols is

$$
\mathcal{S}_{\delta}(M)=\bigoplus_{l=0}^{\infty} \mathcal{S}_{\delta}^{l}(M)
$$

endowed with the classical actions of diffeomorphisms and of vector fields.

2.3. Natural and equivariant quantizations. A quantization on $M$ is a linear bijection $Q_{M}$ from the space of symbols $\mathcal{S}_{\delta}(M)$ to the space of differential operators $\mathcal{D}_{\lambda, \mu}(M)$ such that

$$
\sigma_{l}\left(Q_{M}(S)\right)=S, \forall S \in \mathcal{S}_{\delta}^{l}(M), \forall l \in \mathbb{N},
$$

where $\sigma_{l}$ is the principal symbol operator on the space of operators of order less or equal to $l$.

In the conformal sense, a natural quantization is a collection of quantizations $Q_{M}$ depending on a pseudo-Riemannian metric such that

- For all pseudo-Riemannian metric $g$ on $M, Q_{M}(g)$ is a quantization,

- If $\phi$ is a local diffeomorphism from $M$ to $N$, then one has

$$
Q_{M}\left(\phi^{*} g\right)\left(\phi^{*} S\right)=\phi^{*}\left(Q_{N}(g)(S)\right),
$$

for all pseudo-Riemannian metrics $g$ on $N$, and all $S \in \mathcal{S}_{\delta}(N)$.

Recall now that two pseudo Riemannian metrics $g$ and $g^{\prime}$ on a manifold $M$ are conformally equivalent if and only if there exists a positive function $f$ such that $g^{\prime}=f g$.

A quantization $Q_{M}$ is then conformally equivariant if one has $Q_{M}(g)=$ $Q_{M}\left(g^{\prime}\right)$ whenever $g$ and $g^{\prime}$ are conformally equivalent.

2.4. Conformal group and conformal algebra. These tools were presented in detail in [7, Section 3]. We give here the most important ones for this paper to be self-contained.

Given $p$ and $q$ such that $p+q=m$, we consider the conformal group $G=S O(p+1, q+1)$ and its following subgroup $H$ :

$$
H=\left\{\left(\begin{array}{ccc}
a^{-1} & 0 & 0 \\
a^{-1} A \xi^{\sharp} & A & 0 \\
\frac{1}{2 a}|\xi|^{2} & \xi & a
\end{array}\right): A \in O(p, q), a \in \mathbb{R}_{0}, \xi \in \mathbb{R}^{m *}\right\} /\left\{ \pm I_{m+2}\right\} .
$$

The subgroup $H$ is a semi-direct product $G_{0} \rtimes G_{1}$. Here $G_{0}$ is isomorphic to $C O(p, q)$ and $G_{1}$ is isomorphic to $\mathbb{R}^{m *}$.

The Lie algebra of $G$ is $\mathfrak{g}=s o(p+1, q+1)$. It decomposes as a direct sum of subalgebras :

$$
\mathfrak{g}=\mathfrak{g}_{-1} \oplus \mathfrak{g}_{0} \oplus \mathfrak{g}_{1}
$$

where $\mathfrak{g}_{-1} \cong \mathbb{R}^{m}, \mathfrak{g}_{0} \cong c o(p, q)$, and $\mathfrak{g}_{1} \cong \mathbb{R}^{m *}$. 
This correspondence induces a structure of Lie algebra on $\mathbb{R}^{m} \oplus \operatorname{co}(p, q) \oplus$ $\mathbb{R}^{m *}$. It is easy to see that the adjoint actions of $G_{0}$ and of $\operatorname{co}(p, q)$ on $\mathfrak{g}_{-1}=\mathbb{R}^{m}$ and on $\mathfrak{g}_{1}=\mathbb{R}^{m *}$ coincides with the natural actions of $C O(p, q)$ and of $\operatorname{co}(p, q)$. It is interesting for the sequel to note that :

$$
[v, \xi]=v \otimes \xi+\xi(v) I_{m}-\xi^{\sharp} \otimes v^{b},
$$

if $v \in \mathfrak{g}_{-1}, \xi \in \mathfrak{g}_{1}$ and if $I_{m}$ denotes the identity matrix of dimension $m$. The applications $b$ and $\sharp$ represent the classical isomorphisms between $\mathbb{R}^{m}$ and $\mathbb{R}^{m *}$ detailed in [7].

The Lie algebras corresponding to $G_{0}, G_{1}$ and $H$ are respectively $\mathfrak{g}_{0}, \mathfrak{g}_{1}$, and $\mathfrak{g}_{0} \oplus \mathfrak{g}_{1}$.

2.5. Cartan fiber bundles. It is well-known that there is a bijective and natural correspondence between the conformal structures on $M$ and the reductions of $P^{1} M$ to the structure group $G_{0} \cong C O(p, q)$. The representations $(V, \rho)$ of $G L(m, \mathbb{R})$ defined so far can be restricted to the group $C O(p, q)$. Therefore, once a conformal structure is given, i.e. a reduction $P_{0}$ of $P^{1} M$ to $G_{0}$, we can identify tensors fields of type $V$ as $G_{0}$-equivariant functions on $P_{0}$.

In [4], one shows that it is possible to associate at each $G_{0}$-structure $P_{0}$ a principal $H$-bundle $P$ on $M$, this association being natural and obviously conformally invariant. Since $H$ can be considered as a subgroup of $G_{m}^{2}$, this $H$-bundle can be considered as a reduction of $P^{2} M$. The relationship between conformal structures and reductions of $P^{2} M$ to $H$ is given by the following proposition.

Proposition 1. There is a natural one-to-one correspondence between the conformal equivalence classes of pseudo-Riemannian metrics on $M$ and the reductions of $P^{2} M$ to $H$.

Throughout this work, we will freely identify conformal structures and reductions of $P^{2} M$ to $H$.

2.6. Cartan connections. Let $L$ be a Lie group and $L_{0}$ a closed subgroup. Denote by $\mathfrak{l}$ and $\mathfrak{l}_{0}$ the corresponding Lie algebras. Let $N \rightarrow M$ be a principal $L_{0}$-bundle over $M$, such that $\operatorname{dim} M=\operatorname{dim} L / L_{0}$. A Cartan connection on $N$ is an l-valued one-form $\omega$ on $N$ such that

(1) If $R_{a}$ denotes the right action of $a \in L_{0}$ on $N$, then $R_{a}^{*} \omega=A d\left(a^{-1}\right) \omega$,

(2) If $k^{*}$ is the vertical vector field associated to $k \in \mathfrak{l}_{0}$, then $\omega\left(k^{*}\right)=k$,

(3) $\forall u \in N, \omega_{u}: T_{u} N \mapsto \mathfrak{l}$ is a linear bijection.

When considering in this definition a principal $H$-bundle $P$, and taking as group $L$ the group $G$ and for $L_{0}$ the group $H$, we obtain the definition of Cartan conformal connections.

If $\omega$ is a Cartan connection defined on an $H$-principal bundle $P$, then its curvature $\Omega$ is defined by

$$
\Omega=d \omega+\frac{1}{2}[\omega, \omega]
$$


The notion of Normal Cartan connection is defined by natural conditions imposed on the components of the curvature.

Now, the following result ([4, p. 135]) gives the relationship between conformal structures and Cartan connections :

Proposition 2. A unique normal Cartan conformal connection is associated to every conformal structure $P$. This association is natural.

The connection associated to a conformal structure $P$ is called the normal conformal connection of the conformal structure.

2.7. Lift of equivariant functions. In a previous subsection, we recalled how to associate an $H$-principal bundle $P$ to a conformal structure $P_{0}$. We now recall how the densities and the symbols can be regarded as equivariant functions on $P$.

If $(V, \rho)$ is a representation of $G_{0}$, then we may extend it to a representation $\left(V, \rho^{\prime}\right)$ of $H$ (see [7]). Now, using the representation $\rho^{\prime}$, we can recall the relationship between equivariant functions on $P_{0}$ and equivariant functions on $P$ (see [1]): if we denote by $p$ the projection $P \rightarrow P_{0}$, we have

Proposition 3. If $(V, \rho)$ is a representation of $G_{0}$, then the map

$$
p^{*}: C^{\infty}\left(P_{0}, V\right) \mapsto C^{\infty}(P, V): f \mapsto f \circ p
$$

defines a bijection from $C^{\infty}\left(P_{0}, V\right)_{G_{0}}$ to $C^{\infty}(P, V)_{H}$.

As we continue, we will use the representation $\rho_{*}^{\prime}$ of the Lie algebra of $H$ on $V$. If we recall that this algebra is isomorphic to $\mathfrak{g}_{0} \oplus \mathfrak{g}_{1}$, then we have

$$
\rho_{*}^{\prime}(A, \xi)=\rho_{*}(A), \quad \forall A \in \mathfrak{g}_{0}, \xi \in \mathfrak{g}_{1} .
$$

2.8. The application $Q_{\omega}$. The construction of the application $Q_{\omega}$ is based on the concept of invariant differentiation developed in [1]. Let us recall the definition :

Definition 1. If $f \in C^{\infty}(P, V)$ then $\left(\nabla^{\omega}\right)^{k} f \in C^{\infty}\left(P, \otimes^{k} \mathbb{R}^{m *} \otimes V\right)$ is defined by

$$
\left(\nabla^{\omega}\right)^{k} f(u)\left(X_{1}, \ldots, X_{k}\right)=L_{\omega^{-1}\left(X_{1}\right)} \circ \ldots \circ L_{\omega^{-1}\left(X_{k}\right)} f(u)
$$

for $X_{1}, \ldots, X_{k} \in \mathbb{R}^{m}$.

Definition 2. The map $Q_{\omega}$ is defined by its restrictions to $C^{\infty}\left(P, \otimes^{k} \mathfrak{g}_{-1} \otimes\right.$ $\left.\Delta^{\delta}\left(\mathbb{R}^{m}\right)\right),(k \in \mathbb{N})$ : we set

$$
Q_{\omega}(T)(f)=\left\langle T,\left(\nabla^{\omega}\right)^{k} f\right\rangle
$$

for all $T \in C^{\infty}\left(P, \otimes^{k} \mathfrak{g}_{-1} \otimes \Delta^{\delta}\left(\mathbb{R}^{m}\right)\right)$ and $f \in C^{\infty}\left(P, \Delta^{\lambda}\left(\mathbb{R}^{m}\right)\right)$.

Explicitly, when the symbol $T$ writes $t A \otimes h_{1} \otimes \cdots \otimes h_{k}$ for $t \in C^{\infty}(P)$, $A \in \Delta^{\delta}\left(\mathbb{R}^{m}\right)$ and $h_{1}, \cdots, h_{k} \in \mathbb{R}^{m} \cong \mathfrak{g}_{-1}$ then one has

$$
Q_{\omega}(T) f=t A \circ L_{\omega^{-1}\left(h_{1}\right)} \circ \cdots \circ L_{\omega^{-1}\left(h_{k}\right)} f,
$$

where $t$ is considered as a multiplication operator. 


\subsection{The map $\gamma$.}

Definition 3. We define $\gamma$ on $\otimes^{k} \mathfrak{g}_{-1} \otimes \Delta^{\delta}\left(\mathbb{R}^{m}\right)$ by

$$
\begin{array}{r}
\gamma(h)\left(x_{1} \otimes \cdots \otimes x_{k} \otimes A\right)=\lambda \sum_{i=1}^{k} \operatorname{tr}\left(\left[h, x_{i}\right]\right) x_{1} \otimes \cdots(i) \cdots \otimes x_{k} \otimes A \\
+\sum_{i=1}^{k} \sum_{j>i} x_{1} \otimes \cdots(i) \cdots \otimes \underbrace{\left[\left[h, x_{i}\right], x_{j}\right]}_{(j)} \otimes \cdots \otimes x_{k} \otimes A .
\end{array}
$$

for every $x_{1}, \cdots, x_{k} \in \mathfrak{g}_{-1}, A \in \Delta^{\delta}\left(\mathbb{R}^{m}\right)$ and $h \in \mathfrak{g}_{1}$. Then we extend it to $C^{\infty}\left(P, \otimes^{k} \mathfrak{g}_{-1} \otimes \Delta^{\delta}\left(\mathbb{R}^{m}\right)\right)$ by $C^{\infty}(P)$-linearity.

Definition 4. A trace-free symbol $S$ is a symbol such that $i(g) S=0$ if $g$ is a metric belonging to the conformal structure $P$.

If $S$ is an equivariant function representing a trace-free symbol, $i\left(g_{0}\right) S=0$ if $g_{0}$ represents the canonical metric on $\mathbb{R}^{m}$ corresponding to the conformal structure $P$ (see [7], section 3). It is then easy to show that

Proposition 4. If $S$ is a trace-free symbol of degree $k$, $\gamma(h) S=-k(\lambda m+k-1) i(h) S$. In particular, $\gamma(h) S$ is trace-free.

2.10. Casimir-like operators. Recall that we can define an operator called the Casimir operator $C^{b}$ on $C^{\infty}\left(P, \otimes^{k} \mathfrak{g}_{-1} \otimes \Delta^{\delta}\left(\mathbb{R}^{m}\right)\right)$ (see [7]). This operator $C^{b}$ is semi-simple. The vector space $\otimes^{k} \mathfrak{g}_{-1} \otimes \Delta^{\delta}\left(\mathbb{R}^{m}\right)$ can be decomposed as an $o(p, q)$-representation into irreducible components (since $o(p, q)$ is semisimple):

$$
\otimes^{k} \mathfrak{g}_{-1} \otimes \Delta^{\delta}\left(\mathbb{R}^{m}\right)=\oplus_{s=1}^{n_{k}} I_{k, s} .
$$

The restriction of $C^{b}$ to $C^{\infty}\left(P, I_{k, s}\right)$ is then a scalar multiple of the identity.

We defined in [7] two other operators. If we denote respectively by $\left(e_{1}, \ldots, e_{m}\right)$ and $\left(\epsilon^{1}, \ldots, \epsilon^{m}\right)$ a basis of $\mathfrak{g}_{-1}$ and a basis of $\mathfrak{g}_{1}$ which are dual with respect to the Killing form of $s o(p+1, q+1)$, then

Definition 5. The operator $N^{\omega}$ is defined on $C^{\infty}\left(P, \otimes^{k} \mathfrak{g}_{-1} \otimes \Delta^{\delta}\left(\mathbb{R}^{m}\right)\right)$ by

$$
N^{\omega}=-2 \sum_{i=1}^{m} \gamma\left(\varepsilon^{i}\right) L_{\omega^{-1}}\left(e_{i}\right)
$$

and we set

$$
C^{\omega}:=C^{b}+N^{\omega} .
$$

2.11. Construction of the quantization. Recall that $\otimes^{k} \mathfrak{g}_{-1} \otimes \Delta^{\delta}\left(\mathbb{R}^{m}\right)$ is decomposed as a representation of $o(p, q)$ as the direct sum of irreducible components $I_{k, s}$ with $0 \leq s \leq \frac{k}{2}$ (see [2]). Remark that if $S$ is a trace-free symbol of degree $k$, then $S \in I_{k, 0}$. Denote by $E_{k, s}$ the space $C^{\infty}\left(P, I_{k, s}\right)$ and by $\alpha_{k, s}$ the eigenvalue of $C^{b}$ restricted to $E_{k, s}$.

The tree-like susbspace $\mathcal{T}_{\gamma}\left(I_{k, s}\right)$ associated to $I_{k, s}$ is defined by

$$
\mathcal{T}_{\gamma}\left(I_{k, s}\right)=\bigoplus_{l \in \mathbb{N}} \mathcal{T}_{\gamma}^{l}\left(I_{k, s}\right)
$$


where $\mathcal{T}_{\gamma}^{0}\left(I_{k, s}\right)=I_{k, s}$ and $\mathcal{T}_{\gamma}^{l+1}\left(I_{k, s}\right)=\gamma\left(\mathfrak{g}_{1}\right)\left(\mathcal{T}_{\gamma}^{l}\left(I_{k, s}\right)\right)$, for all $l \in \mathbb{N}$. The space $\mathcal{T}_{\gamma}^{l}\left(E_{k, s}\right)$ is then defined in the same way. Since $\gamma$ is $C^{\infty}(P)$-linear, this space is equal to $C^{\infty}\left(P, \mathcal{T}_{\gamma}^{l}\left(I_{k, s}\right)\right)$.

Definition 6. A value of $\delta$ is critical if there exists $k, s$ such that the eigenvalue $\alpha_{k, s}$ corresponding to an irreducible component $I_{k, s}$ of $\otimes^{k} \mathfrak{g}_{-1} \otimes$ $\Delta^{\delta}\left(\mathbb{R}^{m}\right)$ belongs to the spectrum of the restriction of $C^{b}$ to $\bigoplus_{l \geq 1} \mathcal{T}_{\gamma}^{l}\left(E_{k, s}\right)$.

Recall now the following result :

Theorem 5. If $\delta$ is not critical, for every $T$ in $C^{\infty}\left(P, I_{k, s}\right)$, (where $I_{k, s}$ is an irreducible component of $\otimes^{k} \mathfrak{g}_{-1} \otimes \Delta^{\delta}\left(\mathbb{R}^{m}\right)$ ) there exists a unique function $\hat{T}$ in $C^{\infty}\left(P, \mathcal{T}_{\gamma}\left(I_{k, s}\right)\right)$ such that

$$
\begin{cases}\hat{T} & =T_{k}+\cdots+T_{0}, \quad T_{k}=T \\ C^{\omega}(\hat{T}) & =\alpha_{k, s} \hat{T}\end{cases}
$$

This result allows to define the main ingredient in order to define the quantization : The "modification map", acting on symbols.

Definition 7. Suppose that $\delta$ is not critical. Then the map

$$
R: \oplus_{k=0}^{\infty} C^{\infty}\left(P, S_{\delta}^{k}\right) \rightarrow \oplus_{k=0}^{\infty} C^{\infty}\left(P, \otimes^{k} \mathfrak{g}_{-1} \otimes \Delta^{\delta}\left(\mathbb{R}^{m}\right)\right)
$$

is the linear extension of the association $T \mapsto \hat{T}$.

And finally, the main result :

Theorem 6. If $\delta$ is not critical, then the formula

$$
Q_{M}:(g, T) \mapsto Q_{M}(g, T)(f)=\left(p^{*}\right)^{-1}\left[Q_{\omega}\left(R\left(p^{*} T\right)\right)\left(p^{*} f\right)\right],
$$

(where $Q_{\omega}$ is given by (4)) defines a natural and conformally invariant quantization.

\section{ThE FIRST EXPLICIT FORMULA}

Define now the numbers $\gamma_{2 k-l}$ :

$$
\gamma_{2 k-l}=\frac{m+2 k-l-m \delta}{m} .
$$

We will say that a value of $\delta$ is critical if there are $k, l \in \mathbb{N}$ such that $2 \leq l \leq k+1$ and $\gamma_{2 k-l}=0$.

We can then give the formula giving the natural and conformally invariant quantization in terms of the normal Cartan connection for the trace-free symbols (see [8] for the definitions of $\nabla_{s}^{\omega}$ and Div ${ }^{\omega}$ ) :

Theorem 7. If $\delta$ is not critical, then the collection of maps $Q_{M}: S^{2} T^{*} M \times \mathcal{S}_{\delta}^{k}(M) \rightarrow \mathcal{D}_{\lambda, \mu}(M)$ defined by

$$
Q_{M}(g, S)(f)=p^{*^{-1}}\left(\sum_{l=0}^{k} C_{k, l}\left\langle\operatorname{Div}^{\omega^{l}} p^{*} S, \nabla_{s}^{\omega^{k-l}} p^{*} f\right\rangle\right)
$$


defines a conformally invariant natural quantization for the trace-free symbols if

$$
C_{k, l}=\frac{\left(\lambda+\frac{k-1}{m}\right) \cdots\left(\lambda+\frac{k-l}{m}\right)}{\gamma_{2 k-2} \cdots \gamma_{2 k-l-1}}\left(\begin{array}{c}
k \\
l
\end{array}\right), \forall l \geq 1, \quad C_{k, 0}=1 .
$$

Proof. Thanks to Theorem 5, to the definition of $N^{\omega}$ and to Proposition 4 , one has

$$
S_{l}=\frac{2 \sum_{i=1}^{m} \gamma\left(\varepsilon^{i}\right) L_{\omega^{-1}}\left(e_{i}\right) S_{l+1}}{\alpha_{l, 0}-\alpha_{k, 0}}, \quad 0 \leq l \leq k-1 .
$$

One concludes using Proposition 4 and the fact that (see [2]) :

$$
\alpha_{k, 0}=2 k(1-k+m(\delta-1))-m^{2} \delta(\delta-1) .
$$

Indeed, if $\left(e_{1}, \ldots, e_{m}\right)$ and $\left(\epsilon^{1}, \ldots, \epsilon^{m}\right)$ denote respectively the canonical bases of $\mathbb{R}^{m}$ and $\mathbb{R}^{m *},\left(e_{1}, \ldots, e_{m}\right)$ and $\left(-\epsilon^{1}, \ldots,-\epsilon^{m}\right)$ are Killing-dual with respect to the Killing form given in [2]. One applies eventually Theorem 6.

\section{The SECOND EXPLicit FORMULA}

In order to obtain an explicit formula for the quantization, we need to know the developments of the operators $\nabla^{\omega^{l}}$ and $D i v^{\omega^{l}}$ in terms of operators on $M$.

Let $\gamma$ be a connection on $P_{0}$ corresponding to a covariant derivative $\nabla$ and belonging to the underlying structure of a conformal structure $P$. Recall that $\gamma$ is the Levi-Civita connection of a metric belonging to $P$. We denote by $\tau$ the corresponding function on $P$ with values in $\mathfrak{g}_{1}$, by $\Gamma$ the corresponding deformation tensor (see [1]) and by $\omega$ the normal Cartan connection on $P$.

Let $(V, \rho)$ be a representation of $G_{0}$ inducing a representation $\left(V, \rho_{*}\right)$ of $\mathfrak{g}_{0}$. If we denote by $\rho_{*}^{(l)}$ the canonical representation on $\otimes^{l} \mathfrak{g}_{-1}^{*} \otimes V$ and if $s \in C^{\infty}\left(P_{0}, V\right)_{G_{0}}$, then the development of $\nabla^{\omega^{l}}\left(p^{*} s\right)\left(X_{1}, \ldots, X_{l}\right)$ is obtained inductively as follows (see [1], [8]) :

$$
\begin{aligned}
\nabla^{\omega^{l}}\left(p^{*} s\right)\left(X_{1}\right. & \left., \ldots, X_{l}\right)=\rho_{*}^{(l-1)}\left(\left[X_{l}, \tau\right]\right)\left(\nabla^{\omega^{l-1}}\left(p^{*} s\right)\right)\left(X_{1}, \ldots, X_{l-1}\right) \\
& +S_{\tau}\left(\nabla^{\omega^{l-1}}\left(p^{*} s\right)\right)\left(X_{1}, \ldots, X_{l-1}\right) \\
& +S_{\nabla}\left(\nabla^{\omega^{l-1}}\left(p^{*} s\right)\right)\left(X_{1}, \ldots, X_{l-1}\right) \\
& +S_{\Gamma}\left(\nabla^{\omega^{l-1}}\left(p^{*} s\right)\right)\left(X_{1}, \ldots, X_{l-1}\right) .
\end{aligned}
$$

Recall that $S_{\tau}$ replaces successively each $\tau$ by $-\frac{1}{2}\left[\tau,\left[\tau, X_{l}\right]\right]$, that $S_{\nabla}$ adds successively a covariant derivative on the covariant derivatives of $\Gamma$ and $s$ and that $S_{\Gamma}$ replaces successively each $\tau$ by $\Gamma . X_{l}$.

Recall too that $\Gamma$ is equal in the conformal case to (see [1]) :

$$
\frac{-1}{m-2}\left(\text { Ric }-\frac{g_{0} \mathrm{R}}{2(m-1)}\right)
$$


where Ric and $\mathrm{R}$ denote the equivariant functions on $P$ representing respectively the Ricci tensor and the scalar curvature of the connection $\gamma$.

Proposition 8. If $f \in C^{\infty}\left(P_{0}, \Delta^{\lambda}\left(\mathbb{R}^{m}\right)\right)_{G_{0}}$, then $\nabla^{\omega^{l}}\left(p^{*} f\right)(X, \ldots, X)$ is equal to $g_{0}(X, X) T(X, \ldots, X)$, where $T \in C^{\infty}\left(P, \otimes^{l-2} \mathbb{R}^{m *} \otimes \Delta^{\lambda}\left(\mathbb{R}^{m}\right)\right)$, plus a linear combination of terms of the form

$$
\left(\otimes^{n_{-1}} \tau \otimes p^{*}\left(\otimes^{n_{l-2}} \nabla^{l-2} \Gamma \otimes \ldots \otimes \otimes^{n_{0}} \Gamma \otimes \nabla^{q} f\right)\right)(X, \ldots, X) .
$$

If we denote by $T\left(n_{-1}, \ldots, n_{l-2}, q\right)$ such a term, then $\nabla^{\omega^{l+1}}\left(p^{*} f\right)(X, \ldots, X)$ is equal to the corresponding linear combination of the following sums

$$
\begin{gathered}
\left(-\lambda m-2 l+n_{-1}\right) T\left(n_{-1}+1, \ldots, n_{l-2}, q\right)+T\left(n_{-1}, \ldots, n_{l-2}, q+1\right) \\
+\sum_{j=-1}^{l-2} n_{j} T\left(n_{-1}, \ldots, n_{j}-1, n_{j+1}+1, \ldots, n_{l-2}, q\right)
\end{gathered}
$$

plus $g_{0}(X, X) T^{\prime}(X, \ldots, X)$, where $T^{\prime} \in C^{\infty}\left(P, \otimes^{l-1} \mathbb{R}^{m *} \otimes \Delta^{\lambda}\left(\mathbb{R}^{m}\right)\right)$.

Proof. The proof is similar to the proof of Proposition 7 in [8].

One deduces easily from Proposition 8 the following corollary (see [8] for the definition of $\nabla_{s}$ ):

Proposition 9. If $f \in C^{\infty}\left(P_{0}, \Delta^{\lambda}\left(\mathbb{R}^{m}\right)\right)_{G_{0}}$, then $\nabla_{s}^{\omega^{l}}\left(p^{*} f\right)$ is equal to $g_{0} \vee T$, where $T \in C^{\infty}\left(P, S^{l-2} \mathbb{R}^{m *} \otimes \Delta^{\lambda}\left(\mathbb{R}^{m}\right)\right)$, plus a linear combination of terms of the form

$$
\left(\tau^{n_{-1}} \vee p^{*}\left(\left(\nabla^{l-2} \Gamma\right)^{n_{l-2}} \vee \ldots \vee \Gamma^{n_{0}} \vee \nabla_{s}^{q} f\right)\right)
$$

If we denote by $T\left(n_{-1}, \ldots, n_{l-2}, q\right)$ such a term, then $\nabla_{s}^{\omega^{l+1}}\left(p^{*} f\right)$ is equal to the corresponding linear combination of the following sums

$$
\begin{gathered}
\left(-\lambda m-2 l+n_{-1}\right) T\left(n_{-1}+1, \ldots, n_{l-2}, q\right)+T\left(n_{-1}, \ldots, n_{l-2}, q+1\right) \\
+\sum_{j=-1}^{l-2} n_{j} T\left(n_{-1}, \ldots, n_{j}-1, n_{j+1}+1, \ldots, n_{l-2}, q\right)
\end{gathered}
$$

plus $g_{0} \vee T^{\prime}$, where $T^{\prime} \in C^{\infty}\left(P, S^{l-1} \mathbb{R}^{m *} \otimes \Delta^{\lambda}\left(\mathbb{R}^{m}\right)\right)$.

Proof. The proof is similar to the proof of Proposition 8 in [8].

Remark that the action of the algorithm on the generic term of the part of the development of $\nabla_{s}^{\omega^{l}}\left(p^{*} f\right)$ that does not contain factors $g_{0}$ can be summarized. Indeed, this action gives first

$$
\left(-\lambda m-2 l+n_{-1}\right) T\left(n_{-1}+1, \ldots, n_{l-2}, q\right) .
$$

It gives next

$$
n_{-1} T\left(n_{-1}-1, n_{0}+1, \ldots, n_{l-2}, q\right) .
$$

Finally, it makes act the covariant derivative $\nabla_{s}$ on

$$
\left(\nabla_{s}^{l-2} \Gamma\right)^{n_{l-2}} \vee \ldots \vee \Gamma^{n_{0}} \vee \nabla_{s}^{q} f .
$$


From now, we will denote by $r$ the following multiple of the tensor Ric (recall that Ric is symmetric for a metric connection) :

$$
r:=\frac{1}{(2-m)} \text { Ric. }
$$

In the following proposition, Div denotes the divergence operator :

Proposition 10. If $S \in C^{\infty}\left(P_{0}, \Delta^{\delta} \mathbb{R}^{m} \otimes S^{k} \mathbb{R}^{m}\right)_{G_{0}}$ is trace-free, then Div ${ }^{\omega^{l}}\left(p^{*} S\right)$ is a linear combination of terms of the form

$$
\left\langle\tau^{n_{-1}} \vee p^{*}\left(\left(\nabla_{s}^{k-2} r\right)^{n_{k-2}} \vee \ldots \vee r^{n_{0}}\right), p^{*}\left(\operatorname{Div}^{q} S\right)\right\rangle .
$$

If we denote by $T\left(n_{-1}, \ldots, n_{l-2}, q\right)$ such a term, then $\operatorname{Div}^{\omega} T\left(n_{-1}, \ldots, n_{l-2}, q\right)$ is equal to

$$
\begin{gathered}
\left(\gamma_{2(k-l)-2} m+n_{-1}\right) T\left(n_{-1}+1, \ldots, n_{l-2}, q\right)+T\left(n_{-1}, \ldots, n_{l-2}, q+1\right) \\
+\sum_{j=-1}^{l-2} n_{j} T\left(n_{-1}, \ldots, n_{j}-1, n_{j+1}+1, \ldots, n_{l-2}, q\right) .
\end{gathered}
$$

Proof. The proof is exactly similar to the one of Proposition 9 in [8], using the fact that $S$ and its divergences are trace-free.

Remark that the action of the algorithm on the generic term of the development of $\operatorname{Div}^{\omega^{l}}\left(p^{*} S\right)$ can be summarized. Indeed, this action gives first

$$
\left(\gamma_{2(k-l)-2} m+n_{-1}\right) T\left(n_{-1}+1, \ldots, n_{l-2}, q\right) \text {. }
$$

It gives next

$$
n_{-1} T\left(n_{-1}-1, n_{0}+1, \ldots, n_{l-2}, q\right) .
$$

Finally, it makes act the divergence Div on

$$
\left\langle\left(\nabla_{s}^{k-2} r\right)^{n_{k-2}} \vee \ldots \vee r^{n_{0}}, D i v^{q} S\right\rangle .
$$

Because of the previous propositions, the quantization can be written as a linear combination of terms of the form

$$
\left\langle\left\langle\tau^{n_{-1}} \vee p^{*}\left(\left(\nabla_{s}^{k-2} r\right)^{n_{k-2}} \vee \ldots \vee r^{n_{0}}\right), p^{*}\left(\operatorname{Div}^{q} S\right)\right\rangle, p^{*}\left(\nabla_{s}^{l} f\right)\right\rangle .
$$

In this expression, recall that it suffices to consider the terms for which $n_{-1}=0$ (see [8]).

In the sequel, we will need two operators that we will call $T_{1}$ and $T_{2}$.

If $T$ is a tensor of type $\left(\begin{array}{l}0 \\ j\end{array}\right)$ with values in the $\lambda$-densities, then

$$
T_{1} T=(-\lambda m-j)(j+1) \Gamma \vee T .
$$

If $S$ is a trace-free symbol of degree $j$, then

$$
T_{2} S=\left(m \gamma_{2 k-2}-k+j\right)(k-j+1) i(r) S .
$$

The following results give the explicit developments of $\nabla_{s}^{\omega^{l}}\left(p^{*} f\right)$ and of $\operatorname{Div}^{\omega^{l}}\left(p^{*} S\right)$ : 
Proposition 11. The term of degree $t$ in $\tau$ in the part of the development of $\nabla_{s}^{\omega^{l}}\left(p^{*} f\right)$ that does not contain factors $g_{0}$ is equal to

$$
\left(\begin{array}{l}
l \\
t
\end{array}\right) \prod_{j=1}^{t}(-\lambda m-l+j) p^{*}\left(\pi_{l-t}\left(\sum_{j=0}^{l-t}\left(\nabla_{s}+T_{1}\right)^{j}\right) f\right),
$$

where $\pi_{l-t}$ denotes the projection on the operators of degree $l-t$ (the degree of $\nabla_{s}$ is 1 whereas the degree of $T_{1}$ is 2$)$. We set $\prod_{j=1}^{t}(-\lambda m-l+j)$ equal to 1 if $t=0$.

Proof. The proof is exactly similar to the one of Proposition 10 in [8].

Proposition 12. If $S$ is trace-free, the term of degree $t$ in $\tau$ in the development of $\operatorname{Div}^{\omega^{l}}\left(p^{*} S\right)$ is equal to

$$
\left(\begin{array}{l}
l \\
t
\end{array}\right) \prod_{j=1}^{t}\left(\gamma_{2 k-2} m-l+j\right) p^{*}\left(\pi_{t-l}\left(\sum_{j=0}^{l-t}\left(D i v+T_{2}\right)^{j}\right) S\right),
$$

where $\pi_{t-l}$ denotes the projection on the operators of degree $t-l$ (the degree of Div is -1 whereas the degree of $T_{2}$ is -2$)$. We set the product $\prod_{j=1}^{t}\left(\gamma_{2 k-2} m-l+j\right)$ equal to 1 if $t=0$.

Proof. The proof is completely similar to the one of Proposition 11 in [8].

We can now write the explicit formula giving the natural and conformally invariant quantization for the trace-free symbols :

Theorem 13. The quantization $Q_{M}$ for the trace-free symbols is given by the following formula:

$$
Q_{M}(g, S)(f)=\sum_{l=0}^{k} C_{k, l}\left\langle\pi_{l}\left(\sum_{j=0}^{l}\left(D i v+T_{2}\right)^{j}\right) S, \pi_{k-l}\left(\sum_{j=0}^{k-l}\left(\nabla_{s}+T_{1}\right)^{j}\right) f\right\rangle .
$$

Remark that as $S$ and its divergences are trace-free, one can replace in the definition of the operators $T_{1}$ the deformation tensor $\Gamma$ by $r$. One can easily derive from this formula the formula at the third order. Indeed, if we denote by $D, T, \partial T$ the operators $\nabla_{s}, r \vee$ and $\left(\nabla_{s} r\right) \vee($ resp. Div, $i(r)$ and $\left.i\left(\nabla_{s} r\right)\right)$ and if we denote by $\beta$ the number $-\lambda m$ (resp. $\left.\gamma_{4} m\right)$, one obtains :

$$
\pi_{1}\left(\sum_{j=0}^{1}(D+T)^{j}\right)=D, \quad \pi_{2}\left(\sum_{j=0}^{2}(D+T)^{j}\right)=D^{2}+\beta T
$$

$\pi_{3}\left(\sum_{j=0}^{3}(D+T)^{j}\right)=D^{3}+\beta D T+2(\beta-1) T D=D^{3}+(3 \beta-2) T D+\beta(\partial T)$.

We can then write the formula at the third order :

$$
\begin{gathered}
\left\langle S,\left(\nabla_{s}^{3}-(3 m \lambda+2) r \vee \nabla_{s}-\lambda m\left(\nabla_{s} r\right)\right) f\right\rangle \\
+C_{3,1}\left\langle D i v S,\left(\nabla_{s}^{2}-m \lambda r\right) f\right\rangle+C_{3,2}\left\langle\left(D i v^{2}+m \gamma_{4} i(r)\right) S, \nabla_{s} f\right\rangle
\end{gathered}
$$




$$
+C_{3,3}\left\langle\left(D i v^{3}+\left(3 \gamma_{4} m-2\right) i(r) D i v+m \gamma_{4} i\left(\nabla_{s} r\right)\right) S, f\right\rangle
$$

At the second order, the formula is simply :

$$
\left\langle S,\left(\nabla_{s}^{2}-m \lambda r\right) f\right\rangle+C_{2,1}\left\langle\operatorname{Div} S, \nabla_{s} f\right\rangle+C_{2,2}\left\langle\left(D i v^{2}+m \gamma_{2} i(r)\right) S, f\right\rangle .
$$

\section{ACKNOWLEDGEMENTS}

It is a pleasure to thank $\mathrm{P}$. Mathonet and V. Ovsienko for numerous fruitful discussions and for their interest in our work. We thank the Belgian FNRS for his Research Fellowship.

\section{REFERENCES}

[1] A. Čap, J. Slovák and V. Souček. Invariant operators on manifolds with almost Hermitian symmetric structures. I. Invariant differentiation. Acta Math. Univ. Comenian. (N.S.), 66(1):33-69, 1997.

[2] C. Duval, P. Lecomte and V. Ovsienko. Conformally equivariant quantization : existence and uniqueness. Ann. Inst. Fourier (Grenoble), 49(6):1999-2029, 1999.

[3] M. Eastwood. Higher symmetries of the Laplacian. Ann. of Math. (2) 161 (2005), n3, 1645-1665.

[4] Shoshichi Kobayashi. Transformation groups in differential geometry. SpringerVerlag, New York, 1972. Ergebnisse der Mathematik und ihrer Grenzgebiete, Band 70 .

[5] Pierre B. A. Lecomte. Towards projectively equivariant quantization. Progr. Theoret. Phys. Suppl., (144):125-132, 2001. Noncommutative geometry and string theory (Yokohama, 2001).

[6] P. B. A. Lecomte and V. Yu. Ovsienko. Projectively equivariant symbol calculus. Lett. Math. Phys., 49(3):173-196, 1999.

[7] P. Mathonet and F. Radoux. Existence of natural and conformally invariant quantizations of arbitrary symbols. math.DG 0811.3710.

[8] F. Radoux. Explicit formula for the natural and projectively equivariant quantization. Lett. Math. Phys., 78(2):173-188, 2006. 